\title{
A Note on the Mean Value Theorems
}

\author{
Kung Kuen Tse \\ Department of Mathematics, Kean University, Union, New Jersey, USA \\ Email: ktse@kean.edu
}

How to cite this paper: Tse, K.K. (2021) A

Note on the Mean Value Theorems. $A d$ vances in Pure Mathematics, 11, 395-399. https://doi.org/10.4236/apm.2021.115026

Received: April 4, 2021

Accepted: May 16, 2021

Published: May 19, 2021

\section{Copyright (C) 2021 by author(s) and} Scientific Research Publishing Inc. This work is licensed under the Creative Commons Attribution International License (CC BY 4.0).

http://creativecommons.org/licenses/by/4.0/

\begin{abstract}
The mean value theorem for derivatives says that for a given function over a closed and bounded interval, there is a point $P$ on the graph such that the tangent at $P$ is parallel to the secant through the two endpoints. The mean value theorem for definite integrals says that the area under the function is equal to the area of a rectangle whose base is the length of the interval and height of some point $Q$ on the graph. These two theorems have been studied and utilized extensively and they form the backbone of many important theorems in different branches of mathematics. In this note, we pose the question: for what functions do the two points $P$ and $Q$ always coincide? We find that the only analytic functions satisfying this condition are linear or exponential functions.
\end{abstract}

\section{Keywords}

Mean Value Theorem, Real Analytic Functions, Identity Theorem, Power Series

\section{Introduction}

Let $a<b$. If $f$ is continuous on $[a, b]$ and differentiable on $(a, b)$, then the mean value theorem for derivatives states that [1] [2], there is $c \in(a, b)$ such that

$$
f^{\prime}(c)=\frac{f(b)-f(a)}{b-a} .
$$

If $f$ is continuous on $[a, b]$, the mean value theorem for definite integrals states that there is $c^{*} \in[a, b]$ such that

$$
\int_{a}^{b} f(t) \mathrm{d} t=f\left(c^{*}\right)(b-a) .
$$

Generally speaking, the $c$ in the mean value theorem for the derivative need not equal the $c^{*}$ in the mean value theorem for the definite integral. It is possible that $c=c^{*}$, for example, $f(x)=\mathrm{e}^{x}$. In this case, $c=c^{*}=\ln \left(\frac{\mathrm{e}^{b}-\mathrm{e}^{a}}{b-a}\right)$. In 
this note, we determine all functions for which the two mean value theorems give the same $c$.

Our results rely heavily on the following identity theorem for real analytic functions, its proof can be found in [3].

Theorem If $f$ and $g$ are real analytic functions on an open interval $U$ and there is an open set $W \subset U$ such that

$$
f(x)=g(x), \quad \forall x \in W,
$$

then

$$
f(x)=g(x), \quad \forall x \in U .
$$

\section{Main Result}

Lemma 1 Suppose $f$ is analytic on an open interval $U$, $f$ and $f^{\prime}$ have inverses and for any $a, b \in U$ with $a<b$, there is $c \in(a, b)$ such that

$$
f^{\prime}(c)=\frac{f(b)-f(a)}{b-a} \text { and } \int_{a}^{b} f(t) \mathrm{d} t=f(c)(b-a)
$$

then $f(x)=\alpha \mathrm{e}^{\beta x}+\gamma$, where $\alpha, \beta$ and $\gamma$ are arbitrary real numbers.

Proof. By the hypothesis,

$$
\left(f^{\prime}\right)^{-1}\left(\frac{f(b)-f(a)}{b-a}\right)=c=f^{-1}\left(\frac{\int_{a}^{b} f(t) \mathrm{d} t}{b-a}\right) \text { for all } a<b .
$$

Fix $a \in U$. If we denote $\mathcal{E}_{f}(x)=f^{-1}\left(\frac{\int_{a}^{x} f}{x-a}\right)$, then $f\left(\mathcal{E}_{f}(x)\right)=\frac{\int_{a}^{x} f}{x-a}$ and the above relation becomes

$$
\mathcal{E}_{f}(x)=\mathcal{E}_{f^{\prime}}(x) .
$$

Taking the derivative of both sides, we obtain

$$
\mathcal{E}_{f}^{\prime}(x)=\mathcal{E}_{f^{\prime}}^{\prime}(x) .
$$

To compute $\mathcal{E}_{f}^{\prime}(x)$, we use the derivative of an inverse function and the chain rule,

$$
\begin{aligned}
& \mathcal{E}_{f}^{\prime}(x)=\frac{1}{f^{\prime}\left(f^{-1}\left(\frac{\int_{a}^{x} f}{x-a}\right)\right)} \cdot \frac{\mathrm{d}}{\mathrm{d} x}\left(\frac{\int_{a}^{x} f}{x-a}\right)=\frac{1}{f^{\prime}\left(\mathcal{E}_{f}(x)\right)} \cdot \frac{f(x)(x-a)-\int_{a}^{x} f}{(x-a)^{2}} \\
& =\frac{1}{f^{\prime}\left(\mathcal{E}_{f^{\prime}}(x)\right)} \cdot \frac{f(x)(x-a)-\int_{a}^{x} f}{(x-a)^{2}}=\frac{1}{\frac{\int_{a}^{x} f^{\prime}}{x-a}} \cdot \frac{f(x)(x-a)-\int_{a}^{x} f}{(x-a)^{2}} \\
& =\frac{f(x)-\frac{\int_{a}^{x} f}{x-a}}{f(x)-f(a)} .
\end{aligned}
$$




$$
\mathcal{E}_{f^{\prime}}^{\prime}(x)=\frac{f^{\prime}(x)-\frac{f(x)-f(a)}{x-a}}{f^{\prime}(x)-f^{\prime}(a)} .
$$

We obtain

$$
\frac{f(x)-\frac{\int_{a}^{x} f}{x-a}}{f(x)-f(a)}=\frac{f^{\prime}(x)-\frac{f(x)-f(a)}{x-a}}{f^{\prime}(x)-f^{\prime}(a)}
$$

We need to find $f$ satisfying the above relation. Let $W$ be an open neighborhood of a such that

$$
f(x)=\sum_{n=0}^{\infty} c_{n}(x-a)^{n} \quad \forall x \in W .
$$

Then (1) becomes

$$
\begin{aligned}
& \frac{\left[c_{0}+c_{1}(x-a)+c_{2}(x-a)^{2}+c_{3}(x-a)^{3}+\cdots\right]-\left[c_{0}+\frac{1}{2} c_{1}(x-a)+\frac{1}{3} c_{2}(x-a)^{2}+\frac{1}{4} c_{3}(x-a)^{3}+\cdots\right]}{c_{1}(x-a)+c_{2}(x-a)^{2}+c_{3}(x-a)^{3}+\cdots} \\
& =\frac{\left[c_{1}+2 c_{2}(x-a)+3 c_{3}(x-a)^{2}+4 c_{4}(x-a)^{3}+\cdots\right]-\left[c_{1}+c_{2}(x-a)+c_{3}(x-a)^{2}+c_{4}(x-a)^{3}+\cdots\right]}{2 c_{2}(x-a)+3 c_{3}(x-a)^{2}+4 c_{4}(x-a)^{3}+\cdots}
\end{aligned}
$$

Combining like terms and cross multiplying, we obtain

$$
\begin{aligned}
& {\left[\frac{1}{2} c_{1}+\frac{2}{3} c_{2}(x-a)+\frac{3}{4} c_{3}(x-a)^{2}+\cdots\right]\left[2 c_{2}+3 c_{3}(x-a)+4 c_{4}(x-a)^{2}+\cdots\right]} \\
& =\left[c_{2}+2 c_{3}(x-a)+3 c_{4}(x-a)^{2}+\cdots\right]\left[c_{1}+c_{2}(x-a)+c_{3}(x-a)^{2}+\cdots\right]
\end{aligned}
$$

If $c_{1}=0$, then it is easy to see that $c_{2}=c_{3}=\cdots=0$. In this case, $f(x)=c_{0}$, which cannot happen because we assume that $f$ is invertible. Thus, $c_{1} \neq 0$. Equating the respective coefficients of $x-a$ and $(x-a)^{2}$ on both sides of (2), we obtain

$$
c_{3}=\frac{2}{3} \cdot \frac{c_{2}^{2}}{c_{1}} \text { and } c_{4}=\frac{1}{3} \cdot \frac{c_{2}^{3}}{c_{1}^{2}} \text {. }
$$

In general, for $n \geq 3, c_{n}=\lambda \frac{c_{2}^{n-1}}{c_{1}^{n-2}}$, for some $\lambda$. To find $\lambda$, we observe that $f(x)=\mathrm{e}^{x}$ satisfies (1). Hence the power series of $f(x)=\mathrm{e}^{x}$ should satisfy (2). But in the power series expansion of $f(x)=\mathrm{e}^{x}$ at $x=a$, the coefficient $c_{n}=\frac{e^{a}}{n !}$.

$$
\frac{\mathrm{e}^{a}}{n !}=\lambda \frac{\left(\frac{\mathrm{e}^{a}}{2 !}\right)^{n-1}}{\left(\frac{\mathrm{e}^{a}}{1 !}\right)^{n-2}} \Rightarrow \lambda=\frac{2^{n-1}}{n !}
$$

That is, $c_{n}=\frac{2^{n-1}}{n !} \cdot \frac{c_{2}^{n-1}}{c_{1}^{n-2}}$. In other words, for $f$ to satisfy (1), the power series 
expansion of $f$ at $x=a$ must be

$$
f(x)=c_{0}+c_{1}(x-a)+\sum_{n=2}^{\infty} \frac{2^{n-1}}{n !} \cdot \frac{c_{2}^{n-1}}{c_{1}^{n-2}}(x-a)^{n},
$$

where $c_{0}, c_{1}, c_{2}$ are real numbers. If $c_{2}=0$, then $f(x)=c_{0}+c_{1}(x-a)$, violating the fact that $f^{\prime}$ has an inverse. Thus,

$$
\begin{aligned}
f(x) & =c_{0}+c_{1}(x-a)+\sum_{n=2}^{\infty} \frac{2^{n-1}}{n !} \cdot \frac{c_{2}^{n-1}}{c_{1}^{n-2}}(x-a)^{n} \\
& =c_{0}+c_{1}(x-a)+\frac{c_{1}^{2}}{2 c_{2}} \sum_{n=2}^{\infty} \frac{1}{n !}\left[\frac{2 c_{2}}{c_{1}}(x-a)\right]^{n} \\
& =c_{0}+c_{1}(x-a)+\frac{c_{1}^{2}}{2 c_{2}}\left[\mathrm{e}^{\frac{2 c_{2}}{c_{1}}(x-a)}-1-\frac{2 c_{2}}{c_{1}}(x-a)\right] \\
& =\frac{c_{1}^{2}}{2 c_{2}} \mathrm{e}^{\frac{2 c_{2}}{c_{1}}(x-a)}+\left(c_{0}-\frac{c_{1}^{2}}{2 c_{2}}\right) \\
& =\alpha \mathrm{e}^{\beta(x-a)}+\gamma, \quad \forall x \in W .
\end{aligned}
$$

By the identity theorem, $f(x)=\alpha \mathrm{e}^{\beta x}+\gamma$ on $U$.

Finally, we can relax the requirement that both $f$ and $f^{\prime}$ have inverse.

Theorem 1 If $f$ is analytic on an open interval $U$, and $a, b \in U$ with $a<b$, then the two mean value theorems give the same $c$ if and only if $f(x)=\alpha x+\beta$ or $f(x)=\alpha \mathrm{e}^{\beta x}+\gamma$, where $\alpha, \beta$ and $\gamma$ are any real numbers.

Proof. The necessary condition is obvious. We only prove the sufficient condition. If $f$ is constant on $U$, then we are done. Suppose $f$ is not constant on $U$, then there is some $x_{0} \in U$ such that $f^{\prime}\left(x_{0}\right) \neq 0$. Since $f^{\prime}$ is continuous at $x_{0}$, there is an open interval $I$ of $x_{0}$ with $I \subset U$ such that $f^{\prime} \neq 0$ on $I$. Hence, $f^{-1}$ exists on $I$.

Case 1. $f^{\prime}$ is constant on $I$, then on $I, f(x)=\alpha x+\beta$ for some $\alpha, \beta \in \mathbb{R}$ with $\alpha \neq 0$. By the identity theorem, $f(x)=\alpha x+\beta$ on $U$.

Case $2 f^{\prime}$ is not constant on I, i.e. $f^{\prime \prime}\left(x_{1}\right) \neq 0$ for some $x_{1} \in I$. But $f^{\prime \prime}$ is continuous at $x_{1}$, so there is an open interval $J$ containing $x_{1}$ with $J \subset I$ such that $f^{\prime \prime} \neq 0$ on $J$. Then, both $f$ and $f^{\prime}$ have inverses on $J$, and by the Lemma above, $f(x)=\alpha \mathrm{e}^{\beta x}+\gamma$ on $J$, for some $\alpha, \beta$, and $\gamma \in \mathbb{R}$. But the identity theorem implies that, $f(x)=\alpha \mathrm{e}^{\beta x}+\gamma$ on $U$.

\section{Conclusion}

In this work, we find that if $a<b$ and $f$ is an analytic function, there is $c \in(a, b)$ satisfies both

$$
\int_{a}^{b} f(x) \mathrm{d} x=f(c)(b-a) \text { and } \frac{f(b)-f(a)}{b-a}=f^{\prime}(c)
$$

then $f$ must be a linear function or an exponential function. The proof relies heavily on the fact that $f$ is analytic, we don't know if this condition can be weakened. 


\section{Acknowledgements}

The author is indebted to Alexander Kasiukov for many helpful discussions, without which this project would not be possible

\section{Conflicts of Interest}

The author declares no conflicts of interest regarding the publication of this paper.

\section{References}

[1] Rudin, W. (1976) Principles of Mathematical Analysis. 3rd Edition, McGraw-Hill, New York, 108.

[2] Sahoo, P.K. and Riedel T. (1998) Mean Value Theorems and Functional Equations. World Scientific Publishing Company, Singapore. https://doi.org/10.1142/3857

[3] Krantz, S.G. and Parks, H.R. (2002) A Primer of Real Analytic Functions. 2nd Edition, Birkhäuser, Boston-Basel-Berlin, Corollary 1.2.6., 14.

https://doi.org/10.1007/978-0-8176-8134-0 